\title{
Hybrid synchronous motor electromagnetic torque research
}

\author{
Elena E. Suvorkova ${ }^{a}$, Lev K. Burulko \\ National Research Tomsk Polytechnic University, 634050 Tomsk, Russia
}

\begin{abstract}
Electromagnetic field distribution models in reluctance and permanent magnet parts were made by means of Elcut. Dependences of electromagnetic torque on torque angle were obtained.
\end{abstract}

\section{Introduction}

Present-day position is inextricably connected with developing process in all components of electric drive: electrical machines, power semiconductors and converters on its base.

One of the main electric motors development directions is expanding special motors' construction range for applying in modern competitive object-orientated systems.

Electric machines prospects of promotion lead to its non-traditional usage such as hybrid synchronous motor (HSM). HSM main principles of operation make it possible to use as executive engine in a number of production plants, such as artificial fiber production [1].

Electric drive projecting involves verification and regulation method choice which are determined by main electric machine characteristics. Thus main goals of this paper are hybrid synchronous motor air-gap magnetic field research and electromagnetic torque main parts determination.

\section{Problem statement}

HSM is a machine with stator created on basis of serial asynchronous motor and rotor consisting on two parts. The main rotor part is a reluctance machine which is $70 \%$ of active rotor length [2], the other is synchronous permanent-magnet machine which is $30 \%$ [1]. Power is developed by reluctance machine as the cheapest and simple in design, and energy of constant magnets poles is used for increasing power and improving operational characteristics. That's why we need to obtain electromagnetic torque in both of these parts.

\section{Mathematical model}

Hybrid synchronous motor main part magnetic flux is created by stator's winding reactive current. Running torque arises due to magnetic conductivity difference between axial and quadrature axis and can be determined by equation:

$$
M_{r}=\frac{1}{2} I^{2}\left(x_{d \mathrm{r}}-x_{q \mathrm{r}}\right) \sin 2 \beta=M_{d}-M_{q},
$$

where $\beta$ - angle between stator's winding magnetizing force and rotor pole axis;

$x_{d \mathrm{r}}=\omega \cdot L_{d \mathrm{r}}, x_{q \mathrm{r}}=\omega \cdot L_{q \mathrm{r}}$ - inductive reactances on axial and quadrature axis of HSM reluctance motor part. Thus, running torque of HSM reluctance motor part has two components: $M_{d}$ - component which is coming from magnetic flux on axial axis; $M_{q}-$ component which is coming from magnetic flux on quadrature axis.

Torque of hybrid synchronous motor permanent-magnet part is formed by cooperation of rotating stator magnetic field with embedded magnet's magnetic field and permeance difference on axial and quadrature axis.

However, it is possible to consider that rotating torque component, which is determined by embedded magnets, is a result of total flux interaction with stator winding current in the HSM air-gap. Total flux of permanent-magnet part can be represented in flux vector (geometrical) sum, build by stator winding currents $\Phi_{1}$ and embedded magnet's flux $\Phi_{\mathrm{m}}$ :

$$
\Phi_{\Sigma}=\sqrt{\Phi_{1}^{2}+\Phi_{\mathrm{m}}^{2}+\Phi_{1} \cdot \Phi_{\mathrm{m}} \cos \theta}
$$

where $\theta$ - angle between air-gap flux vectors of hybrid synchronous machine permanent-magnet part.

${ }^{a}$ Corresponding author: suvorkova_elena@mail.ru 
Running torque component $M_{\Sigma}$ coming from total flux is possible to define by magnetic field energy in the air-gap of permanent-magnet part by next equation:

$$
M_{\Sigma}=\frac{d W_{\Sigma}}{d \theta}=\frac{p_{\mathrm{n}} \Phi_{1} \Phi_{\mathrm{m}}}{\mu_{0}} \sin (\theta)
$$

where $W_{\Sigma}$ - total magnetic field energy in the air-gap; $p_{\mathrm{n}}-$ number of pole pares, $\mu_{0}$ - air magnetic permeability.

Thus, HSM permanent-magnet part running torque equation can be written as:

$$
M_{\mathrm{m}}=M_{\Sigma}+M_{d \mathrm{e}}-M_{q \mathrm{e}}
$$

where $M_{d \mathrm{e}}=\frac{1}{2} I^{2} x_{d \mathrm{e}} \sin 2 \theta$ - torque, which was made by axial axis magnetic flux of hybrid synchronous motor permanentmagnet part, $M_{q \mathrm{e}}=\frac{1}{2} I^{2} x_{q \mathrm{e}} \sin 2 \theta$ - torque, which was made by quadrature axis magnetic flux of HSM permanent-magnet part. Then general equation for hybrid machine electromagnetic torque is

$$
M=\frac{1}{2} I^{2}\left(x_{d \mathrm{r}}-x_{q \mathrm{r}}\right) \sin 2 \beta+\frac{p_{\mathrm{n}} \Phi_{1} \Phi_{\mathrm{m}}}{\mu_{0}} \sin (\theta)+M_{d \mathrm{e}}-M_{q \mathrm{e}}
$$

From (5) we can conclude that for machine electromagnetic torque determination it is necessary to know:

- effective stator's winding current $I$;

- inductive parameter values of HSM permanent-magnet and reluctance parts;

- flux values $\Phi_{1}, \Phi_{\mathrm{m}}$ angle values $\beta, \theta$.

\section{Results and discussion}

Hybrid motor air-gap magnetic field model developing by means of ELCUT was done taking as a basis constructional motor specifications: synchronous reluctance part is made as advanced synchronous reluctance motor and permanent-magnet part is synchronous reluctance motor in which big slots magnets $\mathrm{KC}-37 \mathrm{~A}$ are placed[1,2].

Magnetic field distribution in reluctance and permanent-magnet parts of hybrid synchronous motor is shown (Fig.1-2).

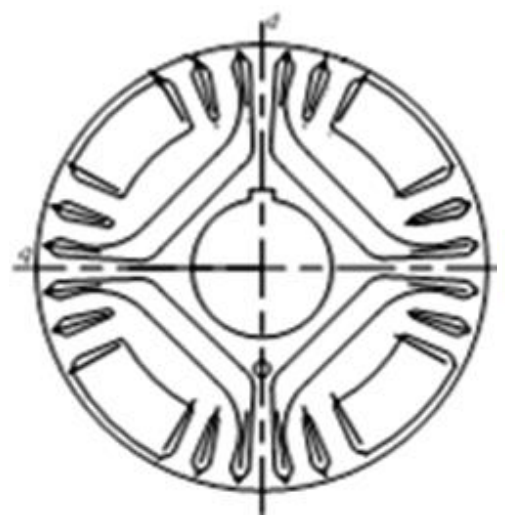

(a)

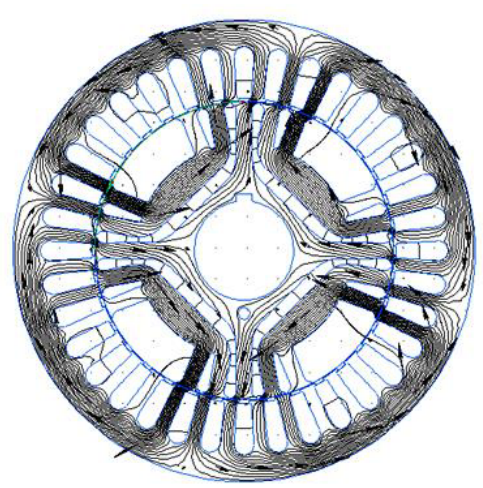

(b)

Figure 1. Magnetic field distribution in relutace part of HSM: (a) synchronous reluctance part cross-section, (b) synchronous reluctance part magnetic flux lines.

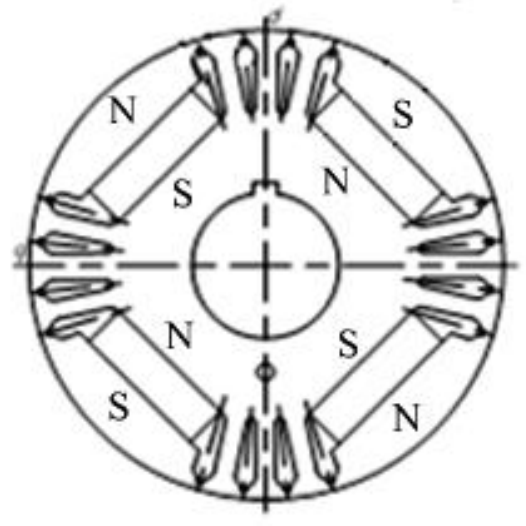

(a)

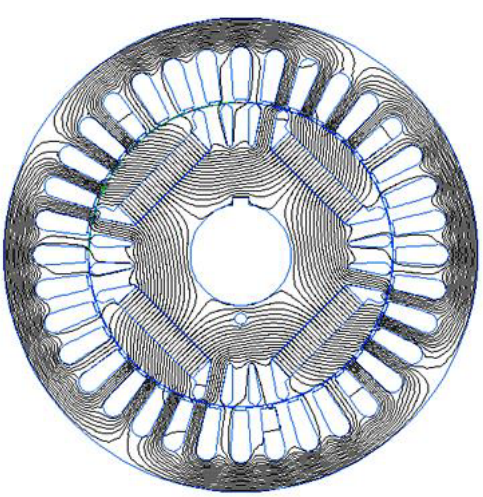

(b) 
Figure 2. Magnetic field distribution in permanent-magnet part of HSM: (a) permanent-magnet part cross-section and rotor's magnets position, (b) permanent-magnet part magnetic flux lines.

Research results showed that permanent-magnet motor part resultant magnetic flux existence is confirmed by magnetic power lines density (Fig.2, b), which provides maximum air-gap induction and additional starting torque of SHM.

By using ELCUT and equation (5) we calculate hybrid machine electromagnetic torque and its components depending on angle between air-gap flux vectors. Electromagnetic torque rating value and its components are shown on (Fig.3).

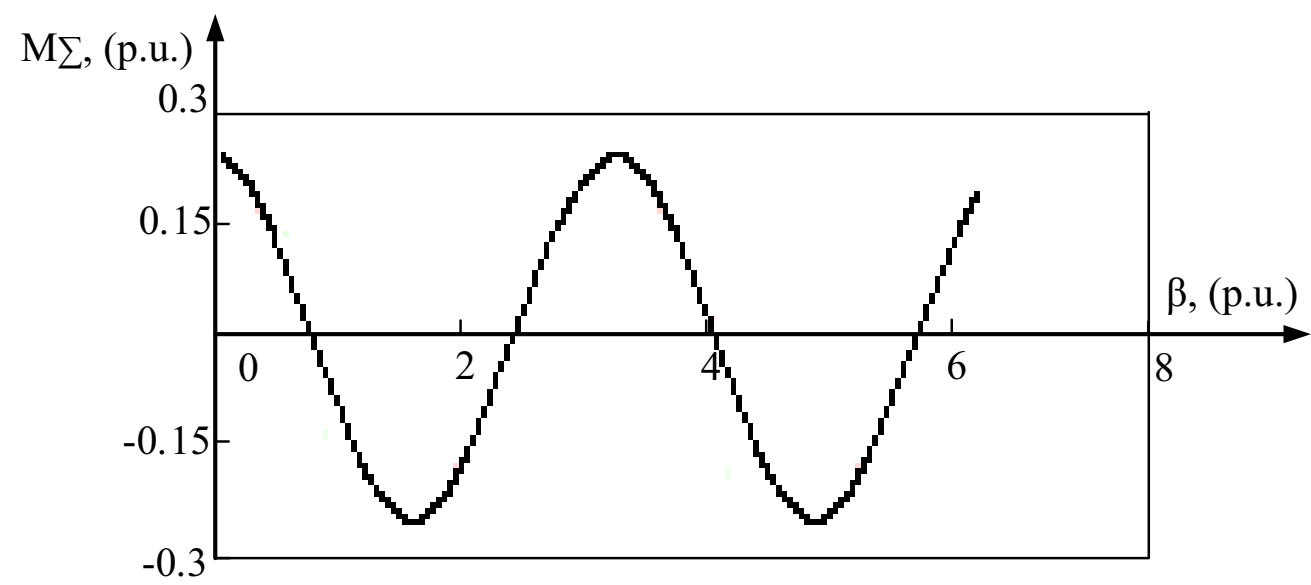

(a)

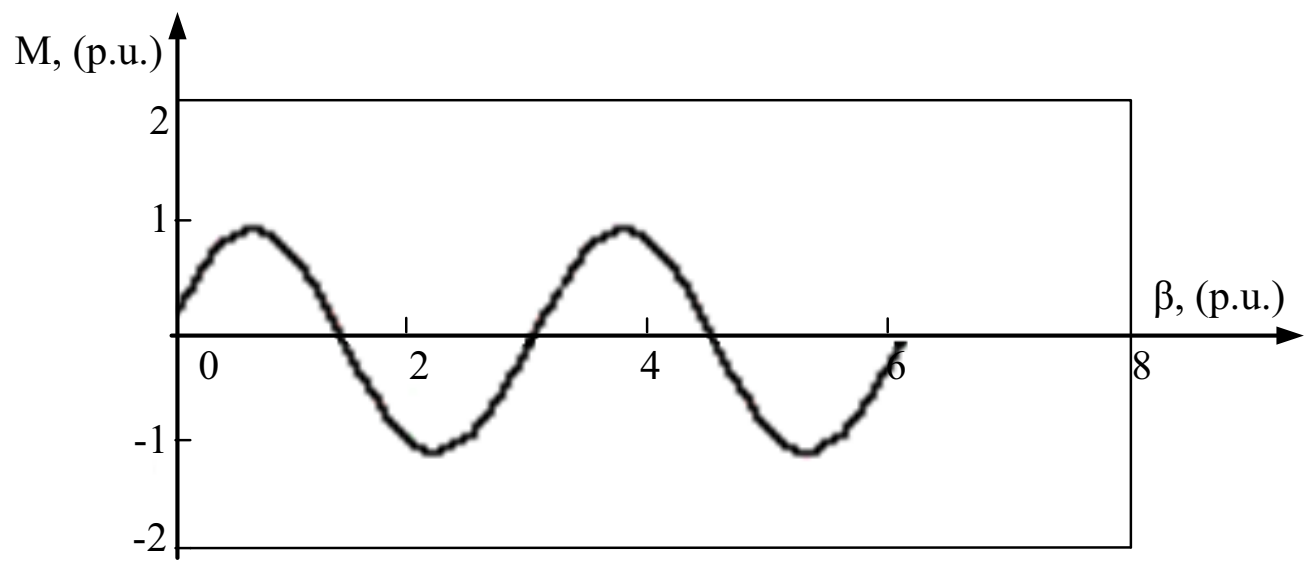

(b)

Figure 3. Electromagnetic torque rating value and its components depending on torque angle : (a) electromagnetic torque in permanent-magnet part of SHM, (b) resultant hybrid synchronous motor electromagnetic torque.

\section{Conclusions}

Resultant electromagnetic flux existence in permanent-magnet part of hybrid synchronous motor is approved by magnetic power lines density (Fig.2) and can give a conclusion about starting torque presence.

Presented calculations (5) which make a dependence (Fig.3,b) also indicate starting torque presence.

\section{References}

1. E.V. Kononenko, "Synchronous reluctance motors", Authors abstract, Tomsk, 1967, pp. 48.

2. A.I. Verhoturov, V.L. Gorobez, A.S. Kuminov, Patient №1663672 (USSR), “The synchronous electric motor with constant magnets", 1991, Bul. № 6.

3. E.E. Suvorkova, I. Vajda, Y.N.Dementiev, A.I. Verhoturov, "Hybrid synchronous motor magnetic field research", The Seventh Japanese-Medeterranean and European Workshop on Applied Electromagnetic Engineering for Magnetic, Superconducting and Nano Materials (JAPMED'7), 2011, pp. 32.

The reported study was supported by the State Task Funding "Science” of Russian Federation (2.1318.2014). 